\title{
Estimation of growths of composite entire and meromorphic functions using their generalized relative orders
}

\author{
Sanjib Kumar Datta ${ }^{1}$, Tanmay Biswas ${ }^{2}$ and Jinarul Haque Shaikh ${ }^{3}$ \\ ${ }^{1}$ Department of Mathematics, University of Kalyani, P.O.-Kalyani, Dist-Nadia, PIN- 741235, West Bengal, India \\ ${ }^{2}$ Rajbari, Rabindrapalli, R. N. Tagore Road, P.O.-Krishnagar, Dist-Nadia, PIN-741101, West Bengal, India \\ ${ }^{2}$ Department of Mathematics, University of Kalyani, P.O.-Kalyani, Dist-Nadia, PIN- 741235, West Bengal, India
}

Received: 6 April 2016, Accepted: 5 June 2016

Published online: 18 June 2016

\begin{abstract}
In this paper we establish some newly developed results related to the growth rates of composite entire and meromorphic functions on the basis of their generalized relative orders and generalized relative lower orders.
\end{abstract}

Keywords: Entire function, meromorphic function, generalized order (generalized lower order), generalized relative order (generalized relative lower order), Property (A), growth.

\section{Introduction}

Let $f$ be a meromorphic and $g$ be an entire function defined in the open complex plane $\mathbb{C}$. The maximum modulus function corresponding to entire $g$ is defined as $M_{g}(r)=\max \{|g(z)|:|z|=r\}$. For meromorphic $f, M_{f}(r)$ can not be defined as $f$ is not analytic. In this situation one may define another function $T_{f}(r)$ known as Nevanlinna's Characteristic function of $f$, playing the same role as maximum modulus function in the following manner:

$$
T_{f}(r)=N_{f}(r)+m_{f}(r),
$$

where the function $N_{f}(r, a)\left(\bar{N}_{f}(r, a)\right)$ known as counting function of $a$-points (distinct $a$-points) of meromorphic $f$ is defined as

$$
\begin{gathered}
N_{f}(r, a)=\int_{0}^{r} \frac{n_{f}(t, a)-n_{f}(0, a)}{t} d t+\bar{n}_{f}(0, a) \log r \\
\left(\bar{N}_{f}(r, a)=\int_{0}^{r} \frac{\bar{n}_{f}(t, a)-\bar{n}_{f}(0, a)}{t} d t+\bar{n}_{f}(0, a) \log r\right),
\end{gathered}
$$

moreover we denote by $n_{f}(r, a)\left(\bar{n}_{f}(r, a)\right)$ the number of $a$-points (distinct $a$-points) of $f$ in $|z| \leq r$ and an $\infty$-point is a pole of $f$. In many occasions $N_{f}(r, \infty)$ and $\bar{N}_{f}(r, \infty)$ are denoted by $N_{f}(r)$ and $\bar{N}_{f}(r)$ respectively. 
Also the function $m_{f}(r, \infty)$ alternatively denoted by $m_{f}(r)$ known as the proximity function of $f$ is defined as follows:

$$
\begin{aligned}
& m_{f}(r)=\frac{1}{2 \pi} \int_{0}^{2 \pi} \log ^{+}\left|f\left(r e^{i \theta}\right)\right| d \theta, \text { where } \\
& \log ^{+} x=\max (\log x, 0) \text { for all } x \geqslant 0 .
\end{aligned}
$$

Also we may denote $m\left(r, \frac{1}{f-a}\right)$ by $m_{f}(r, a)$.

When $f$ is an entire function, the Nevanlinna's Characteristic function $T_{f}(r)$ of $f$ is defined as

$$
T_{f}(r)=m_{f}(r)
$$

However for any two entire functions $f$ and $g$, the ratio $\frac{M_{f}(r)}{M_{g}(r)}$ as $r \rightarrow \infty$ is called the growth of $f$ with respect to $g$ in terms of their maximum moduli. Similarly, when $f$ and $g$ are both meromorphic functions, the ratio $\frac{T_{f}(r)}{T_{g}(r)}$ as $r \rightarrow \infty$ is called the growth of $f$ with respect to $g$ in terms of their Nevanlinna's Characteristic functions. Also the notion of the classical growth indicators such as order and lower order are generally used in computational purpose are defined in terms of their growth with respect to the $\exp z$ function as the following:

$$
\begin{gathered}
\rho_{f}=\limsup _{r \rightarrow \infty} \frac{\log \log M_{f}(r)}{\log \log M_{\exp z}(r)}=\limsup _{r \rightarrow \infty} \frac{\log \log M_{f}(r)}{\log (r)} \\
\left(\lambda_{f}=\liminf _{r \rightarrow \infty} \frac{\log \log M_{f}(r)}{\log \log M_{\exp z}(r)}=\liminf _{r \rightarrow \infty} \frac{\log \log M_{f}(r)}{\log (r)}\right) .
\end{gathered}
$$

If $f$ is a meromorphic function one can easily verify that

$$
\begin{gathered}
\rho_{f}=\limsup _{r \rightarrow \infty} \frac{\log T_{f}(r)}{\log T_{\exp z}(r)}=\limsup _{r \rightarrow \infty} \frac{\log T_{f}(r)}{\log \left(\frac{r}{\pi}\right)}=\limsup _{r \rightarrow \infty} \frac{\log T_{f}(r)}{\log (r)+O(1)} \\
\left(\lambda_{f}=\liminf _{r \rightarrow \infty} \frac{\log T_{f}(r)}{\log T_{\exp z}(r)}=\liminf _{r \rightarrow \infty} \frac{\log T_{f}(r)}{\log \left(\frac{r}{\pi}\right)}=\liminf _{r \rightarrow \infty} \frac{\log T_{f}(r)}{\log (r)+O(1)}\right) .
\end{gathered}
$$

L. Bernal $\{[1],[2]\}$ introduced the relative order between two entire functions to avoid comparing growth just with $\exp z$. Extending the notion of relative order, Lahiri and Banerjee [13] introduced the definition of relative order of a meromorphic function with respect to an entire function.

During the past decades, several researchers have already been exploring their studies in the area of comparative growth properties of composite entire and meromorphic functions in different directions using the classical growth indicators. But at that time, the concepts of relative orders and consequently the generalized relative orders of entire and meromorphic functions with respect to another entire function and as well as their technical advantages of not comparing with the growths of $\exp z$ are not at all known to the researchers of this area. Therefore the studies of the growths of composite entire and meromorphic functions in the light of their relative orders are the prime concern of this paper. In fact some light has already been thrown on such type of works by Datta et al. in [6], [7], [8], [9], [10] and [11]. Actually in this paper we establish some newly developed results related to the growth rates of composite entire and meromorphic functions on the basis of their generalized relative orders (respectively generalized relative lower orders). 


\section{Notation and preliminary remarks}

Our notations are standard within the theory of Nevanlinna's value distribution of entire functions and therefore we do not explain those in detail as available in [12] and [16]. In the sequel the following two notations are used:

$$
\begin{aligned}
\log ^{[k]} x & =\log \left(\log ^{[k-1]} x\right) \text { for } k=1,2,3, \cdots ; \\
\log ^{[0]} x & =x \\
\quad \text { and } & \\
\exp ^{[k]} x & =\exp \left(\exp ^{[k-1]} x\right) \text { for } k=1,2,3, \cdots ; \\
\exp ^{[0]} x & =x .
\end{aligned}
$$

Taking this into account the order (respectively, lower order) of an entire function $f$ is given by

$$
\rho_{f}=\limsup _{r \rightarrow \infty} \frac{\log ^{[2]} M_{f}(r)}{\log r}\left(\text { respectively } \lambda_{f}=\frac{\log ^{[2]} M_{f}(r)}{\log r}\right)
$$

If $f$ is meromrophic, then

$$
\rho_{f}=\limsup _{r \rightarrow \infty} \frac{\log T_{f}(r)}{\log r}\left(\text { respectively } \lambda_{f}=\frac{\log T_{f}(r)}{\log r}\right) .
$$

Let us recall that Sato [14] defined the generalized order and generalized lower order of an entire function $f$, respectively, as follows:

$$
\rho_{f}^{[l]}=\limsup _{r \rightarrow \infty} \frac{\log ^{[l]} M_{f}(r)}{\log r}\left(\text { respectively } \lambda_{f}^{[l]}=\liminf _{r \rightarrow \infty} \frac{\log ^{[l]} M_{f}(r)}{\log r}\right)
$$

where $l$ is any positive integer. These definitions extended the order $\rho_{f}$ and lower order $\lambda_{f}$ of an entire function $f$ since these correspond to the particular case $\rho_{f}^{[2]}=\rho_{f}$ and $\lambda_{f}^{[2]}=\lambda_{f}$.

Similarly, for a meromorphic function $f$, the generalized order and generalized lower order can also be defined as

$$
\rho_{f}^{[l]}=\limsup _{r \rightarrow \infty} \frac{\log ^{[l-1]} T_{f}(r)}{\log r}\left(\text { respectively } \lambda_{f}^{[l]}=\liminf _{r \rightarrow \infty} \frac{\log ^{[l-1]} T_{f}(r)}{\log r}\right)
$$

where $l$ is any positive integer.

Given a non-constant entire function $f$ defined in the open complex plane $\mathbb{C}$, its maximum modulus function $M_{f}(r)$ and Nevanlinna's Characteristic function $T_{f}(r)$ are both strictly increasing and continuous functions of $r$. Also their inverses $M_{f}^{-1}(r):(|f(0)|, \infty) \rightarrow(0, \infty)$ and $T_{f}^{-1}:\left(T_{f}(0), \infty\right) \rightarrow(0, \infty)$ exists respectively and are such that $\lim _{s \rightarrow \infty} M_{f}^{-1}(s)=\infty$ and $\lim _{s \rightarrow \infty} T_{f}^{-1}(s)=\infty$.

In this connection we just recall the following definition which is relevant:

Definition 1. [2] A non-constant entire function $f$ is said have the Property (A) if for any $\sigma>1$ and for all sufficiently large $r,\left[M_{f}(r)\right]^{2} \leq M_{f}\left(r^{\sigma}\right)$ holds. For examples of functions with or without the Property (A), one may see [2]. 
Bernal $\{[1],[2]\}$ introduced the definition of relative order of an entire function $f$ with respect to another entire function $g$, denoted by $\rho_{g}(f)$ to avoid comparing growth just with $\exp z$ as follows:

$$
\rho_{g}(f)=\inf \left\{\mu>0: M_{f}(r)<M_{g}\left(r^{\mu}\right) \text { for all } r>r_{0}(\mu)>0\right\}=\limsup _{r \rightarrow \infty} \frac{\log M_{g}^{-1} M_{f}(r)}{\log r} .
$$

The definition coincides with the classical one [15] if $g(z)=\exp z$.

Similarly, one can define the relative lower order of an entire function $f$ with respect to another entire function $g$ denoted by $\lambda_{g}(f)$ as follows :

$$
\lambda_{g}(f)=\liminf _{r \rightarrow \infty} \frac{\log M_{g}^{-1} M_{f}(r)}{\log r} .
$$

Extending this notion, Lahiri and Banerjee [13] introduced the definition of relative order of a meromorphic function with respect to an entire function in the following way:

Definition 2. [13] Let $f$ be any meromorphic function and $g$ be any entire function. The relative order of $f$ with respect to $g$ is defined as

$$
\rho_{g}(f)=\inf \left\{\mu>0: T_{f}(r)<T_{g}\left(r^{\mu}\right) \text { for all large } r\right\}=\limsup _{r \rightarrow \infty} \frac{\log T_{g}^{-1} T_{f}(r)}{\log r},
$$

Likewise, one can define the relative lower order of a meromorphic function $f$ with respect to an entire function $g$ denoted by $\lambda_{g}(f)$ as follows :

$$
\lambda_{g}(f)=\liminf _{r \rightarrow \infty} \frac{\log T_{g}^{-1} T_{f}(r)}{\log r} .
$$

It is known [13] that if $g(z)=\exp z$ then Definition 2 coincides with the classical definition of the order of a meromorphic function $f$.

Further, Banerjee and Jana [5] gave a more generalized concept of relative order a meromorphic function with respect to an entire function in the following way :

Definition 3. [5] If $l \geq 1$ is a positive integer, then the $l$ - th generalized relative order of a meromorphic function $f$ with respect to an entire function $g$, denoted by $\rho_{g}^{[l]}(f)$ is defined by

$$
\rho_{g}^{[l]}(f)=\limsup _{r \rightarrow \infty} \frac{\log ^{[l]} T_{g}^{-1} T_{f}(r)}{\log r} .
$$

Likewise one can define the generalized relative lower order of a meromorphic function $f$ with respect to an entire function g denoted by $\lambda_{g}^{[l]}(f)$ as

$$
\lambda_{g}^{[l]}(f)=\liminf _{r \rightarrow \infty} \frac{\log ^{[l]} T_{g}^{-1} T_{f}(r)}{\log r} .
$$

\section{Lemmas}

In this section we present some lemmas which will be needed in the sequel. 
Lemma 1. [3] Let $f$ be meromorphic and $g$ be entire then for all sufficiently large values of $r$,

$$
T_{f \circ g}(r) \leqslant\{1+o(1)\} \frac{T_{g}(r)}{\log M_{g}(r)} T_{f}\left(M_{g}(r)\right) .
$$

Lemma 2. [4] Let $f$ be meromorphic and $g$ be entire and suppose that $0<\mu<\rho_{g} \leq \infty$. Then for a sequence of values of $r$ tending to infinity,

$$
T_{f \circ g}(r) \geq T_{f}\left(\exp \left(r^{\mu}\right)\right)
$$

Lemma 3. [11] Let $f$ be an entire function which satisfies the Property (A), $\beta>0, \delta>1$ and $\alpha>2$. Then

$$
\beta T_{f}(r)<T_{f}\left(\alpha r^{\delta}\right) .
$$

\section{Theorems}

In this section we present the main results of the paper.

Theorem 1. Let $f$ be a meromorphic function and $h$ be an entire function with $0<\lambda_{h}^{[l]}(f) \leq \rho_{h}^{[l]}(f)<\infty$ and $g$ be an entire function with $\rho_{g}^{[m]}<\infty$ where $l$ and $m$ are any integers with $l>1$ and $m>2$. If $h$ satisfy the Property $(A)$, then for every positive constant $\mu$ and each $\alpha \in(-\infty, \infty)$,

$$
\lim _{r \rightarrow \infty} \frac{\left\{\log ^{[l]} T_{h}^{-1} T_{f \circ g}(r)\right\}^{1+\alpha}}{\log ^{[l]} T_{h}^{-1} T_{f}\left(\exp ^{[m-1]} r^{\mu}\right)}=0 \text { where } \mu>(1+\alpha) \rho_{g}^{[m]} .
$$

Proof. Let us consider that $\beta>2$ and $\delta>1$. If $1+\alpha \leq 0$, then the theorem is obvious. We consider $1+\alpha>0$.

Since $T_{h}^{-1}(r)$ is an increasing function of $r$, it follows from Lemma 1, Lemma 3 and the inequality $T_{g}(r) \leq \log M_{g}(r)$ \{cf. [5] $\}$ for all sufficiently large values of $r$ that

$$
\begin{aligned}
T_{h}^{-1} T_{f \circ g}(r) & \leqslant T_{h}^{-1}\left[\{1+o(1)\} T_{f}\left(M_{g}(r)\right)\right] \\
T_{h}^{-1} T_{f \circ g}(r) & \leqslant \beta\left[T_{h}^{-1} T_{f}\left(M_{g}(r)\right)\right]^{\delta} \\
\log ^{[l]} T_{h}^{-1} T_{f \circ g}(r) & \leqslant \log ^{[l]} T_{h}^{-1} T_{f}\left(M_{g}(r)\right)+O(1) \\
\log ^{[l]} T_{h}^{-1} T_{f \circ g}(r) & \leqslant\left(\rho_{h}^{[l]}(f)+\varepsilon\right) \exp ^{[m-2]} r^{[m]}+\varepsilon+O(1) .
\end{aligned}
$$

Again for all sufficiently large values of $r$ we get that

$$
\log ^{[l]} T_{h}^{-1} T_{f}\left(\exp ^{[m-1]} r^{\mu}\right) \geq\left(\lambda_{h}^{[l]}(f)-\varepsilon\right) \exp ^{[m-2]} r^{\mu} .
$$

Hence for all sufficiently large values of $r$, we obtain from (1) and (2) that

$$
\frac{\left\{\log ^{[l]} T_{h}^{-1} T_{f \circ g}(r)\right\}^{1+\alpha}}{\log ^{[l]} T_{h}^{-1} T_{f}\left(\exp ^{[m-1]} r^{\mu}\right)} \leq \frac{\left[\left(\rho_{h}^{[l]}(f)+\varepsilon\right) \exp ^{[m-2]} r^{\rho_{g}^{[m]}+\varepsilon}+O(1)\right]^{1+\alpha}}{\left(\lambda_{h}^{[l]}(f)-\varepsilon\right) \exp ^{[m-2]} r^{\mu}}
$$


where we choose $0<\varepsilon<\min \left\{\lambda_{h}^{[l]}(f), \frac{\mu}{1+\alpha}-\rho_{g}^{[m]}\right\}$. So from (3) we obtain that

$$
\lim _{r \rightarrow \infty} \frac{\left\{\log ^{[l]} T_{h}^{-1} T_{f \circ g}(r)\right\}^{1+\alpha}}{\log ^{[l]} T_{h}^{-1} T_{f}\left(\exp ^{[m-1]} r^{\mu}\right)}=0 .
$$

This proves the theorem.

Remark. In Theorem 1 if we take the condition $0<\rho_{h}^{[l]}(f)<\infty$ instead of $0<\lambda_{h}^{[l]}(f) \leq \rho_{h}^{[l]}(f)<\infty$, the theorem remains true with "limit inferior" in place of "limit".

In view of Theorem 1 , the following theorem can be carried out:

Theorem 2. Let $f$ be a meromorphic function and $g, h$ and $k$ be any three entire functions with $\rho_{g}^{[m]}, \lambda_{k}^{[n]}(g)>0$ and $\rho_{h}^{[l]}(f)<\infty$ where $l, m$ and $n$ are any three integers with $l>1, m>2$ and $n>1$. If $h$ satisfies the Property (A), then for every positive constant $\mu$ and each $\alpha \in(-\infty, \infty)$,

$$
\lim _{r \rightarrow \infty} \frac{\left\{\log ^{[l]} T_{h}^{-1} T_{f \circ g}(r)\right\}^{1+\alpha}}{\log ^{[n]} T_{k}^{-1} T_{g}\left(\exp ^{[m-1]} r^{\mu}\right)}=0 \text { where } \mu>(1+\alpha) \rho_{g}^{[m]}
$$

The proof is omitted.

Remark. In Theorem 2 if we take the condition $\rho_{k}^{[n]}(g)>0$ instead of $\lambda_{k}^{[n]}(g)>0$, the theorem remains true with "limit" replaced by "limit inferior".

Theorem 3. Let $f$ be a meromorphic function and $g, h$ be any two entire functions such that $0<\lambda_{h}^{[l]}(f) \leq \rho_{h}^{[l]}(f)<\infty$ and $\lambda_{g}^{[m]}<\mu<\infty$ where $l$ and $m$ are any integers with $l>1$ and $m>2$. Also suppose that $h$ satisfies the Property (A). Then for a sequence of values of $r$ tending to infinity,

$$
\log ^{[l-1]} T_{h}^{-1} T_{f \circ g}(r)<\log ^{[l-1]} T_{h}^{-1} T_{f}\left(\exp ^{[m-1]} r^{\mu}\right) .
$$

Proof. Let us consider $\delta>1$. Since $T_{h}^{-1}(r)$ is an increasing function of $r$, it follows from Lemma 1 that for a sequence of values of $r$ tending to infinity,

$$
\log ^{[l]} T_{h}^{-1} T_{f \circ g}(r) \leqslant\left(\rho_{h}^{[l]}(f)+\varepsilon\right) \exp ^{[m-2]} r^{\lambda_{g}^{[m]}+\varepsilon}+O(1)
$$

Now, from (2) and (4), it follows for a sequence of values of $r$ tending to infinity that

$$
\frac{\log ^{[l]} T_{h}^{-1} T_{f}\left(\exp ^{[m-1]} r^{\mu}\right)}{\log ^{[l]} T_{h}^{-1} T_{f \circ g}(r)} \geq \frac{\left(\lambda_{h}^{[l]}(f)-\varepsilon\right) \exp ^{[m-2]} r^{\mu}}{\left(\rho_{h}^{[l]}(f)+\varepsilon\right) \exp ^{[m-2]} r^{\lambda_{g}^{[m]}+\varepsilon}+O(1)}
$$

As $\lambda_{g}^{[m]}<\mu$ we can choose $\varepsilon(>0)$ in such a way that

$$
\lambda_{g}^{[m]}+\varepsilon<\mu<\rho_{g}^{[m]}
$$

Thus from (5) and (6), we obtain that

$$
\limsup _{r \rightarrow \infty} \frac{\log ^{[l]} T_{h}^{-1} T_{f}\left(\exp ^{[m-1]} r^{\mu}\right)}{\log ^{[l]} T_{h}^{-1} T_{f \circ g}(r)}=\infty
$$


From (7), we obtain for a sequence of values of $r$ tending to infinity and also for $K>1$

$$
\log ^{[l-1]} T_{h}^{-1} T_{f}\left(\exp ^{[m-1]} r^{\mu}\right)>\log ^{[l-1]} T_{h}^{-1} T_{f \circ g}(r) .
$$

Thus the theorem follows. In the line of Theorem 3, we may state the following theorem without proof:

Theorem 4. Let $f$ be a meromorphic function and $g$, $h$ and $k$ be any three entire functions such that $\lambda_{k}^{[n]}(g)>0, \rho_{h}^{[l]}(f)<$ $\infty$ and $\lambda_{g}^{[m]}<\mu<\infty$ where $l, m$ and $n$ are any three integers with $l>1, m>2$ and $n>1$. Also suppose that $h$ satisfies the Property (A), then for a sequence of values of $r$ tending to infinity,

$$
\log ^{[l-1]} T_{h}^{-1} T_{f \circ g}(r)<\log ^{[n-1]} T_{k}^{-1} T_{g}\left(\exp ^{[m-1]} r^{\mu}\right) .
$$

Theorem 5. Let $f$ be a meromorphic function and $h$ be an entire function with $0<\lambda_{h}^{[l]}(f) \leq \rho_{h}^{[l]}(f)<\infty$ where $l$ is any integers with $l>1$. Then for any entire function $g$,

$$
\limsup _{r \rightarrow \infty} \frac{\log ^{[l]} T_{h}^{-1} T_{f \circ g}(r)}{\log ^{[l]} T_{h}^{-1} T_{f}\left(\exp r^{\mu}\right)}=\infty
$$

where $0<\mu<\rho_{g}$.

Proof. Let $0<\mu<\mu^{/}<\rho_{g}$. As $T_{h}^{-1}(r)$ is an increasing function of $r$, it follows from Lemma 2 for a sequence of values of $r$ tending to infinity that

$$
\begin{aligned}
& \log ^{[l]} T_{h}^{-1} T_{f \circ g}(r) \geq \log ^{[l]} T_{h}^{-1} T_{f}\left(\exp \left(r^{\mu /}\right)\right) \\
& \log ^{[l]} T_{h}^{-1} T_{f \circ g}(r) \geq\left(\lambda_{h}^{[l]}(f)-\varepsilon\right) r^{\prime} .
\end{aligned}
$$

Again for all sufficiently large values of $r$ we get that

$$
\log ^{[l]} T_{h}^{-1} T_{f}\left(\exp r^{\mu}\right) \leq\left(\rho_{h}^{[l]}(f)+\varepsilon\right) r^{\mu} .
$$

So combining (8) and (9), we obtain for a sequence of values of $r$ tending to infinity that

$$
\frac{\log ^{[l]} T_{h}^{-1} T_{f \circ g}(r)}{\log ^{[l]} T_{h}^{-1} T_{f}\left(\exp r^{\mu}\right)} \geq \frac{\left(\lambda_{h}^{[l]}(f)-\varepsilon\right) r^{\mu /}}{\left(\rho_{h}^{[l]}(f)+\varepsilon\right) r^{\mu}} .
$$

Since $\mu<\mu^{\prime}$, it follows from (10) that

$$
\limsup _{r \rightarrow \infty} \frac{\log ^{[l]} T_{h}^{-1} T_{f \circ g}(r)}{\log ^{[l]} T_{h}^{-1} T_{f}\left(\exp r^{\mu}\right)}=\infty
$$

Hence the theorem follows.

Corollary 1. Under the assumptions of Theorem 5,

$$
\underset{r \rightarrow \infty}{\limsup } \frac{\log ^{[l-1]} T_{h}^{-1} T_{f \circ g}(r)}{\log ^{[l-1]} T_{h}^{-1} T_{f}\left(\exp r^{\mu}\right)}=\infty, 0<\mu<\rho_{g} .
$$


Proof. In view of Theorem 5, we get for a sequence of values of $r$ tending to infinity that

$$
\begin{aligned}
\log ^{[l]} T_{h}^{-1} T_{f \circ g}(r) & \geq A \log { }^{[l]} T_{h}^{-1} T_{f}\left(\exp r^{\mu}\right), \text { for } A>1 \\
\log ^{[l-1]} T_{h}^{-1} T_{f \circ g}(r) & \geq \log \left\{\log { }^{[l-1]} T_{h}^{-1} T_{f}\left(\exp r^{\mu}\right)\right\}^{A},
\end{aligned}
$$

from which the corollary follows.

Theorem 6. Let $f$ be a meromorphic function and $h, g$ be any two entire functions such that $(i) 0<\lambda_{h}^{[l]}(f) \leq \rho_{h}^{[l]}(f)<\infty$ and $(i i) \lambda_{h}^{[l]}(f \circ g)>0$ where $l$ is any integer with $l>1$. Then

$$
\limsup _{r \rightarrow \infty} \frac{\left[\log { }^{[l]} T_{h}^{-1} T_{f \circ g}(r)\right]^{2}}{\left\{\log ^{[l]} T_{h}^{-1} T_{f}\left(\exp r^{\mu}\right)\right\} \cdot\left\{\log ^{[l]} T_{h}^{-1} T_{f}(r)\right\}}=\infty
$$

where $0<\mu<\rho_{g}$.

Proof. From the definition of relative order and relative lower oder, we obtain for arbitrary positive $\varepsilon$ and for all sufficiently large values of $r$ that

$$
\log ^{[l]} T_{h}^{-1} T_{f \circ g}(r) \geq\left(\lambda_{h}^{[l]}(f \circ g)-\varepsilon\right) \log r
$$

and

$$
\log ^{[l]} T_{h}^{-1} T_{f}(r) \leq\left(\rho_{h}^{[l]}(f)+\varepsilon\right) \log r
$$

Therefore from (12) and (13), it follows for all sufficiently large values of $r$ that

$$
\begin{aligned}
\frac{\log ^{[l]} T_{h}^{-1} T_{f \circ g}(r)}{\log ^{[l]} T_{h}^{-1} T_{f}(r)} \geq \frac{\left(\lambda_{h}^{[l]}(f \circ g)-\varepsilon\right) \log r}{\left(\rho_{h}^{[l]}(f)+\varepsilon\right) \log r} \\
\liminf _{r \rightarrow \infty} \frac{\log ^{[l]} T_{h}^{-1} T_{f \circ g}(r)}{\log ^{[l]} T_{h}^{-1} T_{f}(r)} \geq \frac{\lambda_{h}^{[l]}(f \circ g)}{\rho_{h}^{[l]}(f)} .
\end{aligned}
$$

Thus the theorem follows from (11) and (14).

Similarly one may state the following theorems and corollary without their proofs as those can be carried out in the line of Theorem 5, Theorem 6 and Corollary 1 respectively.

Theorem 7. Let $f$ be a meromorphic function and $h, g$ be any two entire functions with $\lambda_{h}^{[l]}(f)>0$ and $0<\rho_{h}(g)<\infty$ where $l$ is any integer with $l>1$. Then

$$
\limsup _{r \rightarrow \infty} \frac{\log ^{[l]} T_{h}^{-1} T_{f \circ g}(r)}{\log T_{k}^{-1} T_{g}\left(\exp r^{\mu}\right)}=\infty
$$

where $0<\mu<\rho_{g}$.

Theorem 8. Let $f$ be a meromorphic function and $h, g$ be any two entire functions such that $(i) 0<\rho_{k}(g)<\infty$, (ii) $\lambda_{h}^{[l]}(f)>0$ and (iii) $\lambda_{h}^{[l]}(f \circ g)>0$ where $l$ is any integer with $l>1$. Then

$$
\limsup _{r \rightarrow \infty} \frac{\left[\log { }^{[l]} T_{h}^{-1} T_{f \circ g}(r)\right]^{2}}{\left\{\log T_{k}^{-1} T_{g}\left(\exp r^{\mu}\right)\right\} \cdot\left\{\log T_{k}^{-1} T_{g}(r)\right\}}=\infty
$$

where $0<\mu<\rho_{g}$. 
Corollary 2. Under the assumptions of Theorem 7,

$$
\limsup _{r \rightarrow \infty} \frac{\log ^{[l-1]} T_{h}^{-1} T_{f \circ g}(r)}{T_{k}^{-1} T_{g}\left(\exp r^{\mu}\right)}=\infty, 0<\mu<\rho_{g} .
$$

Theorem 9. Let $f$ be a meromorphic function and $h$ be an entire function with $0<\lambda_{h}^{[l]}(f) \leq \rho_{h}^{[l]}(f)<\infty$ where $l$ is any integers with $l>1$. Then for any entire function $g$,

$$
\underset{r \rightarrow \infty}{\limsup } \frac{\log ^{[l+1]} T_{h}^{-1} T_{f \circ g}\left(\exp r^{A}\right)}{\log ^{[l+1]} T_{h}^{-1} T_{f}\left(\exp r^{\mu}\right)}=\infty,
$$

where $0<\mu<\rho_{g}$ and $A>0$.

Proof. Let $0<\mu^{/}<\rho_{g}$. As $T_{h}^{-1}(r)$ is an increasing function of $r$, it follows from (8) for a sequence of values of $r$ tending to infinity that

$$
\log ^{[l+1]} T_{h}^{-1} T_{f \circ g}(r) \geq O(1)+\mu^{\prime} \log r .
$$

So for a sequence of values of $r$ tending to infinity, we get from above that

$$
\log ^{[l+1]} T_{h}^{-1} T_{f \circ g}\left(\exp r^{A}\right) \geq O(1)+\mu^{/} r^{A} .
$$

Again we have for all sufficiently large values of $r$ that

$$
\log ^{[l+1]} T_{h}^{-1} T_{f}\left(\exp r^{\mu}\right) \leq O(1)+\mu \log r .
$$

Now combining (15) and (16), we obtain for a sequence of values of $r$ tending to infinity that

$$
\frac{\log ^{[l+1]} T_{h}^{-1} T_{f \circ g}\left(\exp r^{A}\right)}{\log ^{[l+1]} T_{h}^{-1} T_{f}\left(\exp r^{\mu}\right)} \geq \frac{O(1)+\mu^{/} r^{A}}{O(1)+\mu \log r}
$$

from which the theorem follows.

In view of Theorem 9, the following theorem can be carried out:

Theorem 10. Let $f$ be a meromorphic function and $h, k, g$ be any three entire functions with $\lambda_{h}^{[l]}(f)>0$ and $0<\rho_{k}(g)<\infty$ where $l$ is any integer with $l>1$. Then

$$
\limsup _{r \rightarrow \infty} \frac{\log ^{[l+1]} T_{h}^{-1} T_{f \circ g}\left(\exp r^{A}\right)}{\log ^{[2]} T_{k}^{-1} T_{g}\left(\exp r^{\mu}\right)}=\infty,
$$

where $0<\mu<\rho_{g}$ and $A>0$.

The proof is omitted.

Theorem 11. Let $h$ be an entire function satisfying the Property $(A)$ and $p$ be a meromorphic function such that $\lambda_{h}^{[l]}(p)>0$. Also let $g$ and $k$ be any two entire with finite non zero order and such that $\rho_{g}<\rho_{k}$. Then for every meromorphic function $f$ with $0<\rho_{h}^{[l]}(f)<\infty$,

$$
\limsup _{r \rightarrow \infty} \frac{\log ^{[l]} T_{h}^{-1} T_{p \circ k}(r)}{\log ^{[l]} T_{h}^{-1} T_{f \circ g}(r)+\log ^{[l]} T_{h}^{-1} T_{f}(r)}=\infty
$$

where $l$ is any integer with $l>1$. 
Proof. Since $\rho_{g}<\rho_{k}$, we can choose $\varepsilon(>0)$ in such a way that

$$
\rho_{g}+\varepsilon<\mu<\rho_{k}-\varepsilon
$$

As $T_{h}^{-1}(r)$ is an increasing function of $r$, it follows from Lemma 2 for a sequence of values of $r$ tending to infinity that

$$
\begin{gathered}
\log ^{[l]} T_{h}^{-1} T_{p \circ k}(r) \geq \log ^{[l]} T_{h}^{-1} T_{p}\left(\exp r^{\mu}\right) \text { where } 0<\mu<\rho_{k} \leq \infty \\
\text { i.e., } \log ^{[l]} T_{h}^{-1} T_{p \circ k}(r) \geq\left(\lambda_{h}^{[l]}(p)-\varepsilon\right) r^{\mu} .
\end{gathered}
$$

Now from the definition of relative order of $f$ with respect to $l$, we have for arbitrary positive $\varepsilon$ and for all sufficiently large values of $r$ that

$$
\log ^{[l]} T_{h}^{-1} T_{f}(r) \leq\left(\rho_{h}^{[l]}(f)+\varepsilon\right) \log r .
$$

Now for any $\delta>1$, we get from (1), (18), (19) and in view of (17) for a sequence of values of $r$ tending to infinity that

$$
\begin{gathered}
\frac{\log ^{[l]} T_{h}^{-1} T_{p \circ k}(r)}{\log ^{[l]} T_{h}^{-1} T_{f \circ g}(r)+\log ^{[l]} T_{h}^{-1} T_{f}(r)} \geq \frac{\left(\lambda_{h}^{[l]}(p)-\varepsilon\right) r^{\mu}}{\left(\rho_{h}^{[l]}(f)+\varepsilon\right) r^{\rho_{g}+\varepsilon}+\left(\rho_{h}^{[l]}(f)+\varepsilon\right) \log r+O(1)} \\
\frac{\log ^{[l]} T_{h}^{-1} T_{p \circ k}(r)}{\log ^{[l]} T_{h}^{-1} T_{f \circ g}(r)+\log ^{[l]} T_{h}^{-1} T_{f}(r)}=\infty,
\end{gathered}
$$

which proves the theorem.

In the line of Theorem 11 the following theorem can be carried out:

Theorem 12. Let h be an entire function satisfying the Property $(A)$ and p be a meromorphic function such that $\lambda_{h}^{[l]}(p)>0$. Also let $q, g$ and $k$ be any three entire functions such that $0<\rho_{g}<\rho_{k}<\infty$ and $\rho_{q}(g)<\infty$. Then for every meromorphic function $f$ with $0<\rho_{h}^{[l]}(f)<\infty$,

$$
\limsup _{r \rightarrow \infty} \frac{\log ^{[l]} T_{h}^{-1} T_{p \circ k}(r)}{\log ^{[l]} T_{h}^{-1} T_{f \circ g}(r)+\log T_{q}^{-1} T_{g}(r)}=\infty
$$

where $l$ is any integer with $l>1$.

\section{References}

[1] L. Bernal : Crecimiento relativo de funciones enteras. Contribución al estudio de lasfunciones enteras coníndice exponencial finito, Doctoral Dissertation, University of Seville, Spain, 1984.

[2] L. Bernal : Orden relative de crecimiento de funciones enteras, Collect. Math., Vol. 39 (1988), pp.209-229.

[3] W. Bergweiler : On the Nevanlinna characteristic of a composite function, Complex Variables, Vol. 10 (1988), pp. $225-236$.

[4] W. Bergweiler : On the growth rate of composite meromorphic functions, Complex Variables, Vol. 14 (1990), pp. 187-196.

[5] D. Banerjee and S. Jana : Meromorphic functions of relative order $(p, q)$, Soochow Journal of Mathematics, Vol. 33, No. 3 (July, 2007), pp. 343-357.

[6] S. K. Datta and T. Biswas : Growth of entire functions based on relative order, Int. J. Pure Appl. Math., Vol. 51, No. 1 (2009), pp. 49-58. 
[7] S. K. Datta and T. Biswas : Relative order of composite entire functions and some related growth properties, Bull. Cal. Math. Soc., Vol.102, No.3 (2010) pp.259-266.

[8] S. K. Datta , T. Biswas and R. Biswas : On relative order based growth estimates of entire functions, International J. of Math. Sci. \& Engg. Appls. (IJMSEA), Vol. 7, No. II (March, 2013), pp. 59-67.

[9] S. K. Datta , T. Biswas and R. Biswas : Comparative growth properties of composite entire functions in the light of their relative order, The Mathematics Student, Vol. 82, No. 1-4 (2013), pp. 209-216.

[10] S. K. Datta, T. Biswas and C. Biswas: Growth analysis of composite entire and meromorphic functions in the light of their relative orders, International Scholarly Research Notices, Volume 2014, Article ID538327, 6 pages; http://dx.doi.org/10.1155/2014/538327.

[11] S. K. Datta, T. Biswas and C. Biswas: Measure of growth ratios of composite entire and meromorphic functions with a focus on relative order, International J. of Math. Sci. \& Engg. Appls. (IJMSEA), Vol. 8 No. IV (July, 2014), pp. 207-218.

[12] W. K. Hayman : Meromorphic Functions, The Clarendon Press, Oxford (1964).

[13] B. K. Lahiri and D. Banerjee : Relative order of entire and meromorphic functions, Proc. Nat. Acad. Sci. India Ser. A., Vol. 69(A), No. 3, (1999), pp.339-354.

[14] D. Sato : On the rate of growth of entire functions of fast growth, Bull. Amer. Math. Soc., Vol. 69 (1963), pp. 411-414.

[15] E. C. Titchmarsh : The theory of functions, 2nd edition, Oxford University Press, Oxford, 1939.

[16] G. Valiron : Lectures on the general theory of integral functions, Chelsea Publishing Company, 1949. 